Ind. Health, 1967, 5, 206.

\title{
EVALUATION METHODS FOR VIBRATION EFFECTS
}

\author{
PART 2. MEASUREMENT OF EQUAL SENSATION LEVEL \\ FOR WHOLE BODY BETWEEN VERTICAL AND \\ HORIZONTAL SINUSOIDAL VIBRATIONS
}

\author{
Toshisuke MIWA
}

National Institute of Industrial Health, Kizuki-Sumiyoshi, Kawasaki

(Received November 27, 1967)

\begin{abstract}
The sensation of horizontal vibration was equated to that of vertical vibration of the same frequency at $5,10,20,30,60,100,200$ and $300 \mathrm{c} / \mathrm{s}$. The sensation of vertical vibration above $5 \mathrm{c} / \mathrm{s}$ was found to be stronger than that of horizontal vibration by 10 $\mathrm{dB}$ for sitting and by $13 \mathrm{~dB}$ for standing.

Then, the horizontal vibrations from 0.5 to $200 \mathrm{c} / \mathrm{s}$ were equated in sensation with the vertical vibration of $20 \mathrm{c} / \mathrm{s}$ at $30 \mathrm{~dB}$ (vibration acceleration level (VAL)) for sitting alone. The contours obtained in this experiment and the curves of the equal sensation for horizontal vibration on whole body are quite similar with each other except that VAL at the standard frequency $(20 \mathrm{c} / \mathrm{s})$ of the latter is larger by $10 \mathrm{~dB}$ than that of the former.
\end{abstract}

The threshold and equal sensation contours for evaluation of vibration effect on human body were described in the previous report (Part 1), in which each vibration of vertical and horizontal directions for sitting and standing from 0.5 to $300 \mathrm{c} / \mathrm{s}$ was equated in sensation to the standard vibration at $20 \mathrm{c} / \mathrm{s}$. Now, the study on a mutual relation of sensation between both vibrations will serve the understanding of the following problems.

First, various vibrations in actual environments are usually mixed vibrations of vertical and horizontal directions. If the mutual relation is known, the effect of mixed vibrations on human body will be estimated by the effect of either direction of both vibrations. Secondary, the equal sensation contours for vertical and horizontal vibrations are completely superposed with each other above $7 \mathrm{c} / \mathrm{s}$ (Fig 13 in Part 1). But whether two vibrations in this frequency band are equally felt in sensation is questionable. This problem will also be solved by that mutual relation.

The papers which treated experimentally of this mutual relation problem could not be found. In this report, the mutual relation was obtained by simultaneously using vertical and horizontal vibration tables. 


\section{Procedure}

The experiments were done by two electro-dynamic vibration tables. The vertical vibration table had the size of $200 \times 250 \times 20 \mathrm{~mm}^{3}$ and the supporting part of this table was constructed by two cantilevers at interval of $300 \mathrm{~mm}$, each of which was made of two sheets of hard leaf spring $\left(200 \times 65 \times 2 \mathrm{~mm}^{3}\right)$ of phosphorus bronze (Fig. 1, 5). The power amplifier was an usual vacuum tube type with an output transformer. This table had the frequency band ranged from 5 to $500 \mathrm{c} / \mathrm{s}$ and amplitude limit of $\pm 0.5 \mathrm{~cm}$ in vibration displacement at $5 \mathrm{c} / \mathrm{s}$ and of $10 \mathrm{~g}$ in vibration acceleration at high frequency $\left(1 \mathrm{~g}=980 \mathrm{~cm} / \mathrm{sec}^{2}\right)$.

For the horizontal vibration, the same table as in the previous report was used. Both vibration tables were checked never to excite the undesirable vibration such as rocking motion etc.

This experiment was proceeded by the paired comparisons' method and the intensities of vibration signals were given the subjects in ascending and descending series. Ten subjects who were the same members as in Part 1 were seated on the vertical vibration table first. The tester intermittently gave the subject the vibration with a certain amplitude three times by exchanging the step of the switch every $3 \mathrm{sec}$ in order to memorize the vibration sensation as standard. The vertical vibration was adopted as the standard against the horizontal vibration. Then, the subjects were ridden on the horizontal vibration table and the horizontal vibration with variable amplitude were given by the same procedure to compare with the vertical vibration sensation memorized previously. These paird comparisons were repeated till the subject could get the equal sensation between the vibrations of two directions by changing the intensity of horizontal vibration in ascending and descending series, in which the average of the levels of the horizontal vibration observed in two series was got as the equal sensation levels. The vibration intensities of both directions were changed with the two attenuators controlled by the tester and calibrated by optic means at several low frequencies. In ascending series, the horizontal vibration was begun at lower level in comparison with the vertical vibration as the standard. While, in descending series, the horizontal vibration was started from more higher level by $6 \mathrm{~dB}$ than the equated level in ascending series.

About the frequency, the experiment was carried out in the following two procedures. In the first experiment, the frequencies of both vibrations were chosen to be the same. For example, the horizontal vibration at $60 \mathrm{c} / \mathrm{s}$ was compared with the vertical one at $60 \mathrm{c} / \mathrm{s}$ and so on. The frequencies were varied to 5, 10, 20, 60, 100, 200 and $300 \mathrm{c} / \mathrm{s}$. Especially, the experiment at $20 \mathrm{c} / \mathrm{s}$ seems to be fundamental, since this frequency was used as the standard in the series of these studies. The subjects were tested in two postures, sitting erect and standing erect.

In the second experiment, the horizontal vibration at each frequency from 
T. MIWA

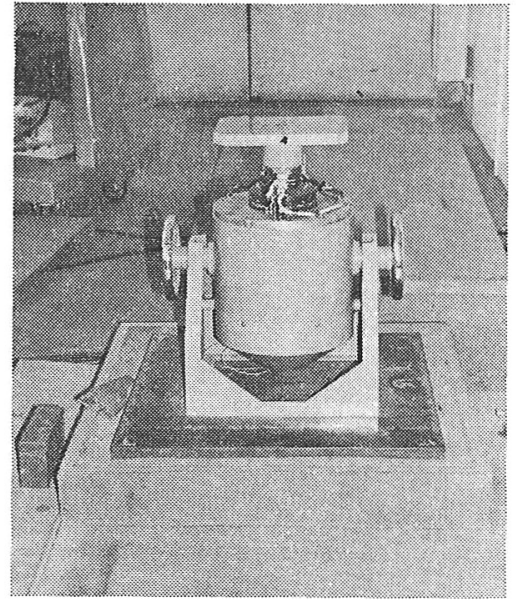

1

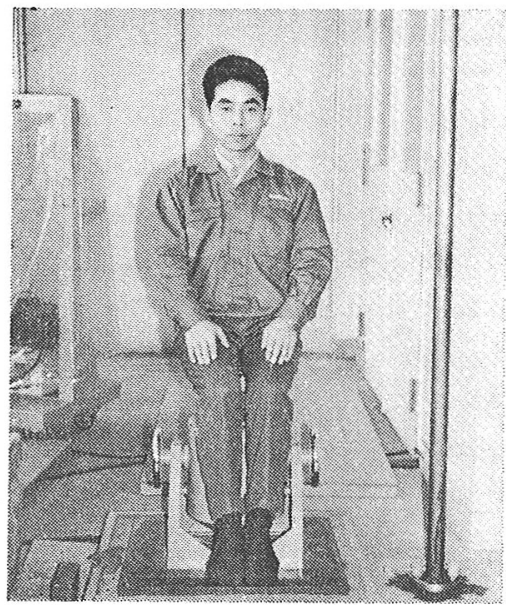

3
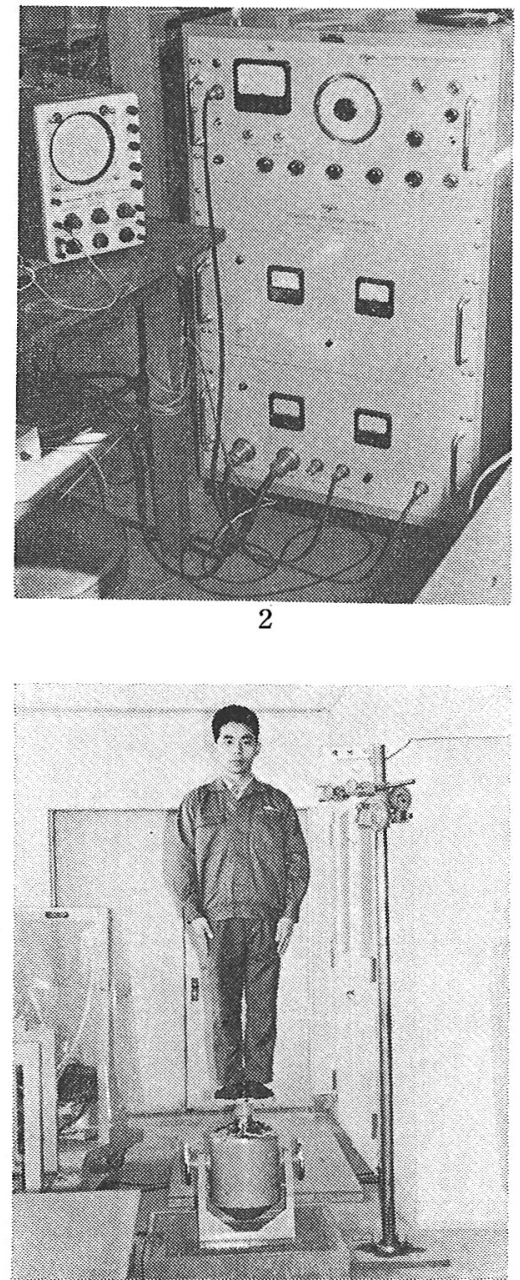

4

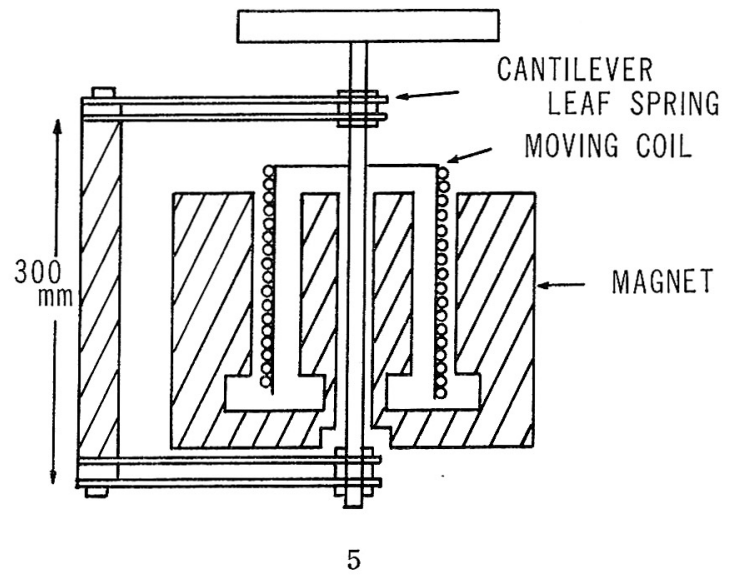

Fig. 1. Vertical vibration tables, its control amplifier and postures of subject for sitting and standing. 


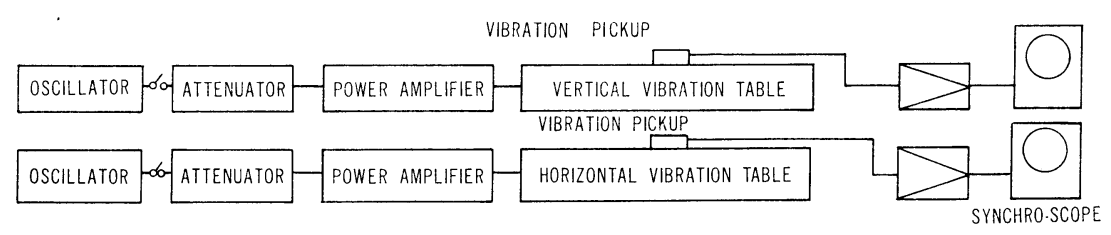

Fig. 2. Block diagram for measurement.

0.5 to $300 \mathrm{c} / \mathrm{s}$ was equated in sensation to the vertical vibration with $30 \mathrm{~dB}$ (VAL) at $20 \mathrm{c} / \mathrm{s}$ selected as standard. In this case, the sitting posture alone was tested, because the results for standing could be predicted from the first experiment. The reason why the vertical vibration was adopted as standard is that the subjects showed better discrimination for the vibration sensation in this direction than in the horizontal direction.

The photographs of the vibration tables and the postures of the subjects are shown in Fig. 1 and the block diagram for these measurements is also indicated in Fig. 2.

\section{Results AND Discussions}

In Figs. 3, 4, 5 and 6, the average values and the standard deviations of 10 men $\left(\sigma=1.028 \sqrt{\frac{1}{n-1} \sum\left(x_{i}-\bar{x}\right)^{2}} ; \mathrm{n}=10\right)$ on the seven frequencies are indicated. The vibration was measured in vibration acceleration level (VAL) defined in Part 1. Ordinate is taken as VAL of horizontal vibration. Abscissa is VAL of vertical vibration in Figs. 3, 4 and 5, and the frequency (c/s) in Fig. 6.

In the first experiment, the vertical and horizontal vibrations were compared with each other at the same frequency. The results obtained from the postures of sitting and standing erect at $20 \mathrm{c} / \mathrm{s}$ are shown in Fig. 3. The linear relation was obtained from this figure,

$$
\mathrm{VAL}_{\mathrm{h}}=\mathrm{VAL}_{\mathrm{v}}+\mathrm{C}
$$

Where $\mathrm{VAL}_{\mathrm{h}}$; vibration acceleration level(VAL) of horizontal vibration, $\mathrm{VAL}_{\mathrm{v}} ; \mathrm{VAL}$ of vertical vibration, $\mathrm{C}$; constant value (10 $\mathrm{dB}$ for sitting or $13 \mathrm{~dB}$ for standing).

Then, the same experiments at the other frequencies, 5, 10,60, 100, 200 and $300 \mathrm{c} / \mathrm{s}$, were tried and the results for two postures are shown in Figs. 4 and 5 respectively. The values of $\mathrm{C}$ are approximately equal to that at $20 \mathrm{c} / \mathrm{s}$ except small deviation at 200 and $300 \mathrm{c} / \mathrm{s}$ for standing.

From these results, the sensation of vertical vibration is stronger than that of horizontal vibration at each frequency over all ranges $(5-300 \mathrm{c} / \mathrm{s})$ by about 10 $\mathrm{dB}$ for sitting and $13 \mathrm{~dB}$ for standing

The standard deviation of pooled data of this experiment is $\pm 1 \mathrm{~dB}$. The judgment of equal sensation between both vibrations is considered to be sharp enough and so, these two sensations seem to be convertible. In the second expe- 


\section{T. MIWA}

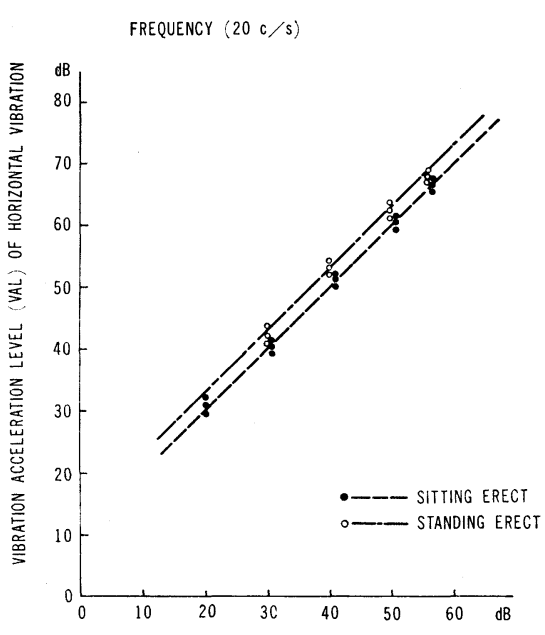

VIBRATION ACCELERATION LEVEL VAL) OF VERTICAL VIBRATION

Fig. 3. The horizontal vibration was equated in sensation to the vertical vibration in the same frequency at $20 \mathrm{c} / \mathrm{s}$.

In Figs. 3, 4, 5 and 6, the average values and the standrd deviations of 10 men on the seven frequencies are indicated by various signs, for example, $\bigcirc, \odot, \Delta, \times, \bigcirc, \vee$ and The center of three signs arranged lengthwise shows the average value and both outside signs the standard deviation.

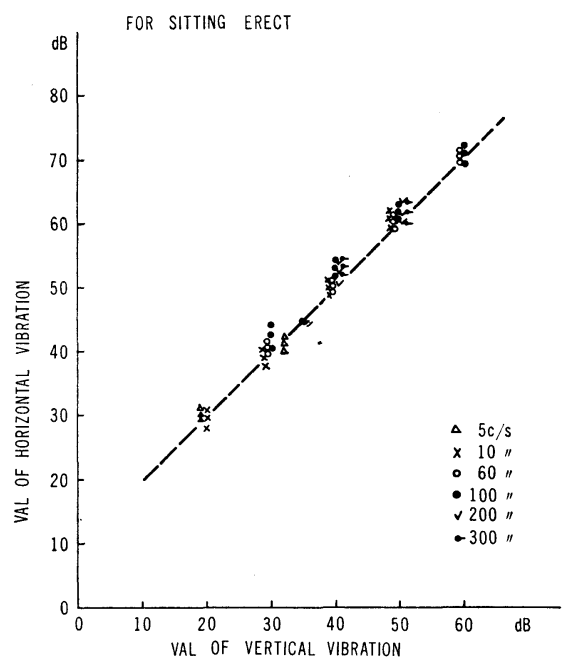

Fig. 4. The horizontal vibration was equated in sensation to the vertical vibration in the same frequencies at $5,10,60,100,200$ and $300 \mathrm{c} / \mathrm{s}$ for sitting erect.

riment, the horizontal vibration from 0.5 to $300 \mathrm{c} / \mathrm{s}$ were equated in sensation to the vertical vibration at $20 \mathrm{c} / \mathrm{s}$ for the sitting erect alone. The average value and standard deviation with 10 men are shown in Fig. 6 and the sign of star (30 $\mathrm{dB}$ at $20 \mathrm{c} / \mathrm{s}$ ) the standard level of the vertical vibration. The curve connected with these average values was composed of three bending lines and two bending 


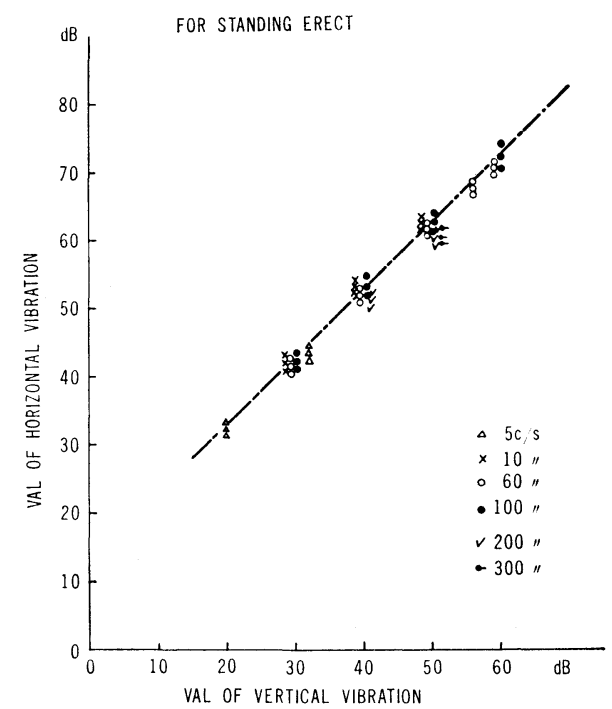

Fig. 5. The horizontal vibration was equated in sensation to the vertical vibration in the same frequency at $5,10,60,100,200$ and $300 \mathrm{c} / \mathrm{s}$ for standing erect.

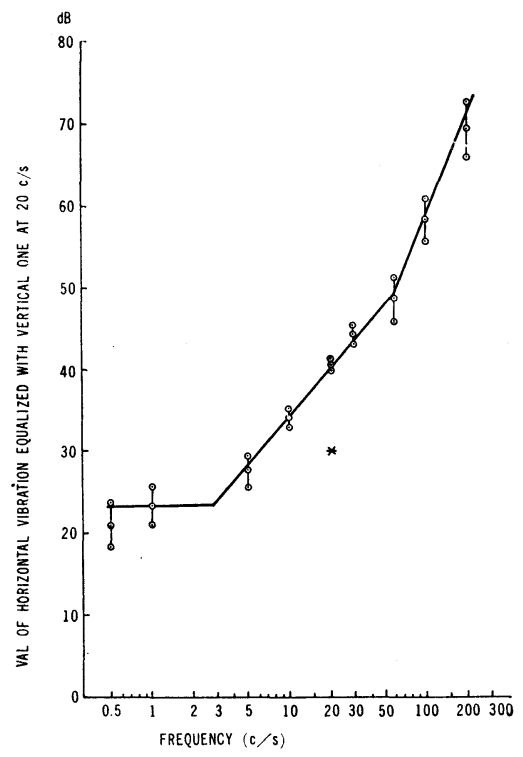

Fig. 6. The horizontal vibration from 0.5 to $200 \mathrm{c} / \mathrm{s}$ was equated in sensation to the standard vertical vibration at $20 \mathrm{c} / \mathrm{s}$ with $30 \mathrm{VAL}$ (vibration acceleration level).

points, 3 and $60 \mathrm{c} / \mathrm{s}$. This curve and the equal sensation contours for horizontal vibration as shown in Fig. 13 in Part 1 were in satisfactory agreement with each other in their shapes. This case, in which the sensation of horizontal vibration, $0.5-200 \mathrm{c} / \mathrm{s}$, was directly equated to the vertical vibration with $30 \mathrm{~dB}$ (VAL) at $20 \mathrm{c} / \mathrm{s}$, gave the similar result that the sensation of horizontal vibration $(0.5-300$ $\mathrm{c} / \mathrm{s})$ was first projected to that of the horizontal vibration at $20 \mathrm{c} / \mathrm{s}$, and then the 


\section{T. MIWA}

sensation of horizontal and vertical vibrations at the same frequency $20 \mathrm{c} / \mathrm{s}$ was equated with each other. This fact is very interesting problem concerning the projection of one vibration frequency to another in sensation and also seems to prove the correctness of Fig. 13 in Part 1 by having reexamined the same item from the other viewpoints.

The standard deviation was small near the standard frequency $(20 \mathrm{c} / \mathrm{s})$ and increased with the frequency being apart from $20 \mathrm{c} / \mathrm{s}$. This tendency is explaned by easiness of the judgment of equal sensation near the standard frequency. The sensation of horizontal vibration is convertible to vertical one with the difference of 10 or $13 \mathrm{~dB}$ for sitting or standing respectively, and this seems to depend upon the essential difference of perceptional mechanism for vibration. It is worthy of notice that this difference of 10 or $13 \mathrm{~dB}$ approximately coincided with the difference of the threshold between both vibrations for sitting or standing shown in Fig. 13 in Part 1.

On the other hand, when the hand sensation for horizontal vibration was equated to that for vertical vibration. the difference between vertical and horizontal vibration was not found as shown in the next paper. And also, the difference of threshold value between them was not observed.

These two facts show there is a certain relation between the difference of threshold and the value of the equal sensation conversion for both vibrations.

The difference of conversion value between sitting and standing $(13-10=3 \mathrm{~dB})$ may be explained by that of vibration transmission in human body. For example, the vertical vibration in standing erect is very well conducted by spinal column from heel to brain because of vibration characteristics of framework.

The difference between erect and relaxed postures was not observed in preliminary experiments.

Thus, in Fig. 13 in Part 1, the sensation of the vertical vibration on the equal sensation curve should be equated to that of horizontal vibration with more higher level by $10 \mathrm{VGL}$ (vibration greatness level) for sitting. For example, above $5 \mathrm{c} / \mathrm{s}$, the sensation of the vertical vibration on the curve of $20 \mathrm{VGL}$ may be equated that of the horizontal vibration on the curve of $30 \mathrm{VGL}$. Under $5 \mathrm{c} / \mathrm{s}$, as the curves of $20 \mathrm{VGL}$ for vertical vibration and $30 \mathrm{VGL}$ for horizontal vibration are in agreement with each other in Fig. 13, the sensation of both vibrations with the same vibration acceleration level may be similar for sitting. For standing, the horizontal vibration level should be shifted up by 33 VGL. 